\title{
Contribution to the Sand Fly Fauna (Diptera: Phlebotominae) of Rio Grande do Sul, Brazil and Leishmania (Viannia) Infections
}

\author{
Onilda Santos da Silva ${ }^{+}$, Jörg Grunewald
}

Institute of Tropical Medicine, University of Tuebingen, Wilhelmstrasse 27, D-72074 Tuebingen, Germany

American cutaneous leishmaniasis (ACL) is endemic in the State of Rio Grande do Sul, Brazil. As reports of nearby hospitals suggest, the Parque Estadual do Turvo seems to serve as a source for the disease. During three months from November 1996 to February 1997 we collected, in this park, 2,228 sand flies (10 Lutzomyia species and 2 Brumptomyia species). We applied the polymerase chain reaction to 920 females which belong to the following species: Lu. migonei, Lu. pessoai, Lu. fischeri, Lu. misionensis, Lu. lanei, Lu. neivai, Lu. shannoni, and Lu. monticola, in an attempt to verify natural infection by Leishmania (Viannia), the causative agent of ACL. Le. (Viannia) infections were demonstrated by DNA amplification from two Lu. pessoai and one Lu. misionensis female. Lu. pessoai have been found with leptomonas in the gut believed to be Le. (V.) braziliensis in other endemic areas of northeastern and southeastern Brazil. However, Lu. misionensis has never been found carrying a natural infection of Le. (Viannia).

Key words: sand flies - Phlebotominae - Leishmania (Viannia) - polymerase chain reaction - southern Brazil

American cutaneous leishmaniasis (ACL) is endemic in the State of Rio Grande do Sul, Brazil (Forattinni 1973). There are some notified cases of the disease (Lacerda 1994). Hospitals in the area confirmed some cases of cutaneous and mucocutaneous leishmaniasis every year. All patients infected with the disease originated from rural areas of the Parque Estadual do Turvo's marginal forest zone. The park seems to serve as a source for human Leishmania infection.

The disease does not seem to be a public health problem therefore the phlebotomine fauna and the dynamics of transmission in this area are still unkown. There is only one report on the biogeographical distribution of phlebotomine species in this area (Dias et al. 1997). Nevertheless, forest clearing and agricultural activities may result in an increase in the number of leishmaniasis cases in the area.

The purpose of the present study was to determine the phlebotomine species of certain areas in the park, and to identify the vector species and their infection rate with Le. (Viannia) using the polymerase chain reaction (PCR).

\footnotetext{
${ }^{+}$Corresponding author. Fax: +49-7071-295486. E-mail: silva-onilda@gmx.de

Received 25 September 1998

Accepted 15 June 1999
}

\section{MATERIALS AND METHODS}

Study area - The Parque Estadual do Turvo is located in the north of Rio Grande do Sul $\left(27^{\circ} 20^{\prime}\right.$ $27^{\circ} 10^{\prime} \mathrm{S}$ and $54^{\circ} 10^{\prime}-53^{\circ} 40^{\prime} \mathrm{W}$ ). The region represents the most important primary rain forest in southern Brazil and corresponds to an untouched natural ecosystem. It has a wet and hot subtropical climate, with an annual rainfall greater than 1,900 $\mathrm{mm}$. The study was conducted at the Salto Yucumã tourist lodge which sits on the edge of the forest, and is managed by a local family. At the lodge, domestic animals such as cattle, chicken, cats and dogs can be found.

Collection and identification of Phlebotominae - During three months (November 1996 to February 1997) sand flies were caught at weekly intervals. Three ecologically different areas at the lodge were examined: domicile, peridomicile, and forest. The domicile area corresponded to the inside and outside walls of the house, while the peridomicile area was delineated by the walls of the cattle stable and chicken pen. The collection area in the forest was located within a distance of $3 \mathrm{~km}$ from the lodge. Sand flies were caught with a manual aspirator in domicile and peridomicile areas, while in the forest, manual aspirators as well as a Shannon trap (Shannon 1939) were used. The specimens were stored in 70\% ethylalcohol and brought to the laboratory in Tübingen, Germany for processing. Identification of the sand flies was carried out according to the classification schemes of Young and Duncan (1994) and Martins et al. 
(1978). Females were identified based on the shape of the spermathecae and the number of cibarial teeth and a portion was preserved on slides using Entellan medium. Males were identified by the shape of their genitalia and also preserved on slides using Entellan medium.

Infection rate of the collected sand flies with Le. (Viannia) - PCR was employed to verify if any of 920 females, which belonged to eight species were naturally infected by Le. (Viannia), the causative agent of ACL. Two oligonucleotide primers (GAACGGGGTTTCTGTATGC and TACTCCCC GACATGCCTCTG) were employed to amplify a 70 bp sequence of the conserved region of $L e$. (Viannia) minicircle kinetoplast. Le. (V.) braziliensis $\mathrm{MHOM} / \mathrm{BR} / 75 / \mathrm{M} 2903$ served as positive control while Le. mexicana MNYC/BZ/62/ M379 was used as the negative. The methods used to apply the PCR were adapted from Arevalo et al. (1993) with some modifications.

After morphological identification the females were immersed individually in $50 \mu \mathrm{l}$ lysis buffer (Tris- $\mathrm{HCl} 1 \mathrm{M}$ pH 8.0, EDTA 0.5M pH 8.0, SDS $1 \%$ ) containing Proteinase $\mathrm{K}$ and incubated at $65^{\circ} \mathrm{C}$ for $3 \mathrm{hr}$ for DNA extraction. The PCR master mix was prepared with $5 \mathrm{U} / \mu \mathrm{l}$ of Taq Polymerase, 2.5 $\mathrm{mM}$ from each of dATP, dCTP, dTTP, dGTP, 70 $\mathrm{mM}$ Tris- $\mathrm{HCl} 0.5 \mathrm{M}(\mathrm{pH} 8.8), 17 \mathrm{mM}\left(\mathrm{NH}_{4}\right)_{2} \mathrm{SO}_{4}$, $0.1 \mathrm{mM}$ B-Mercaptoethanol, $2 \mathrm{mM} \mathrm{MgCl} 2$, and 1 $\mu \mathrm{M}$ of each oligonucleotide. For the amplification $5 \mu \mathrm{l}$ of DNA from a pool of 10 females was added to $15 \mu \mathrm{l}$ of the above-described solution.

The PCR master mix product was prepared shortly before the samples were transferred into the PCR machine, giving better results than using a product that has been previously frozen. Amplified products were analyzed by electrophoresis at $65 \mathrm{~V}$ in NEEO (Roth) agarose gel (3\%), stained with ethidium bromide and visualized under UV light.

\section{RESULTS}

Phlebotominae fauna - A total of 2,220 Lutzomyia specimens and eight Brumptomyia specimens were collected and identified. More sand flies were collected in the peridomicile area $(1,306 /$ $58.6 \%)$ than in the domicile $(648 / 29.1 \%)$ and forest areas $(274 / 12.3 \%)$. We were able to identify 10 species from the Lutzomyia genus and two from the genus Brumptomyia. Regarding Lutzomyia, 18 specimens could not be identified (Table). The predominant species was $L u$. migonei (domicile $47.8 \%$ and peridomicile $62.2 \%$ ) followed by $L u$. pessoai, Lu. fischeri, Lu. misionensis, Lu. lanei, Lu. neivai, Lu. shannoni, and Lu. monticola. All species are known to take blood meals on humans. In the forest, all of the above mentioned species were found, with $L u$. misionensis as the predominant species (32.5\%) followed by $\mathrm{Lu}$. monticola (16.8\%), Lu. lanei (14.9\%) and Lu. shannoni (14.9\%). In addition, the species Lu. correalimai, Lu. schreiberi, as well as Brump-tomyia pintoi and B. nitzulescui were found in the forest but these species did not occur in the other two areas. The proportion of males was highest in the domicile and peridomicile areas $(60.8 \%)$, the females dominated in the forest $(85 \%)$.

Infection rate - A total of 920 females was analyzed for Le. (Viannia) infection. The species were: Lu. migonei (200), Lu. pessoai (360), Lu. fischeri

TABLE

Distribution of Lutzomyia and Brumptomyia species in Parque Estadual doTurvo from November 1996 to February 1997

\begin{tabular}{|c|c|c|c|c|c|c|c|c|}
\hline \multirow[t]{2}{*}{ Species } & \multicolumn{2}{|c|}{ Domicile } & \multicolumn{2}{|c|}{ Peridomicile } & \multicolumn{2}{|c|}{ Forest } & \multirow[t]{2}{*}{ Total } & \multirow[t]{2}{*}{$\%$} \\
\hline & Female & Male & Female & Male & Female & Male & & \\
\hline Lu. migonei & 106 & 204 & 131 & 681 & 5 & 7 & 1,134 & 50.9 \\
\hline Lu. pessoai & 154 & 5 & 196 & 20 & 14 & - & 389 & 17.5 \\
\hline Lu. lanei & 36 & 46 & 63 & 26 & 22 & 19 & 212 & 9.5 \\
\hline Lu. neivai & 13 & 48 & 41 & 67 & 2 & 2 & 173 & 7.8 \\
\hline Lu. misionensis & 9 & - & 32 & - & 89 & - & 130 & 5.8 \\
\hline Lu. shannoni & 8 & - & 27 & - & 41 & - & 76 & 3.4 \\
\hline Lu. monticola & - & - & 18 & - & 45 & 1 & 64 & 2.9 \\
\hline Lu. fischeri & 17 & - & - & - & 3 & - & 20 & 0.9 \\
\hline Lu. schreiberi & - & - & - & - & - & 2 & 2 & 0.1 \\
\hline Lu. correalimai & - & - & - & - & - & 2 & 2 & 0.1 \\
\hline$L u$. spp. & 2 & - & 4 & - & 12 & - & 18 & 0.8 \\
\hline B. pintoi & - & - & - & - & - & 4 & 4 & 0.2 \\
\hline B. nitzulescui & - & - & - & - & - & 4 & 4 & 0.2 \\
\hline Total & 345 & 303 & 512 & 794 & 233 & 41 & 2,228 & 100 \\
\hline
\end{tabular}


(10), Lu. misionensis (120), Lu. lanei (90), Lu. neivai (40), Lu. shannoni (50), and Lu. monticola (50). Only two (0.6\%) Lu. pessoai females and one $(0.8 \%) \mathrm{Lu}$. misionensis female were found positive for 70bp of Le. (Viannia) DNA. Lu. pessoai infected females were collected in the domicile area, and Lu. misionensis in the forest (Figure).

\section{DISCUSSION}

Five species that were isolated in the park, $L u$. migonei, Lu. pessoai, Lu. fischeri, Lu. neivai and $L u$. shannoni, are suspected to be vectors of $L e$. (Viannia) elsewhere in Brazil. They have been found infected with promastigotes in foci of tegumentary leishmaniasis (Young \& Duncan 1994 and others).

Two species, Lu. schreiberi and B. pintoi, have never been isolated in the area before.

Lu. misionensis was found to be the predominant species in the forest, while Lu. migonei and $L u$. pessoai were the predominant species in domicile and peridomicile areas. In contrast, Dias et al. (1997), who studied the sand fly fauna in the park during January to February 1986, identified Lu. fisheri and Lu. intermedia as the prevalent species in the forest.

Only $L u$. pessoai and $L u$. misionensis were found to be infected with Le. (Viannia). In the case of Lu. misionensis this is the first report of a natural infection with Le. (Viannia). Lu. pessoai is considered as a probable vector of Le. (Viannia) in other endemic areas of northeastern and southeastern Brazil.

Infected females were identified in the domicile area and the forest. The infection rate, however, was very low, being $0.6 \%$ for $L u$. pessoai and $0.8 \%$ for $L u$. misionensis respectively. Thus, these two vector species do not appear to play an important role in the transmission of leishmaniasis infection to humans. The infection rate of the total of 920 females investigated was only $0.3 \%$, and in fact the infection of humans with leishmaniasis is rare in the area. In comparison, in Mexico, in the the State of Campeche, an endemic ACL area, Rebollar-Téllez et al. (1996) found an infection rate of $2.8 \%$ by dissecting 1,288 Lutzomyia females.

It is important that further collections of sand flies be made in different ecological areas of the park and in different seasons of the year to obtain more information on the significance of ACL and the dynamics of its transmission in and around the park.

\section{ACKNOWLEDGEMENTS}

To Prof. Dr Wolf Engels and Prof. Dr Klaus Hartfelder from Tuebingen University in connection with the Pró-Araucária Project for providing travel funds. To Prof. Dr Carlos Brisola Marcondes from Universidade Federal de Santa Catarina for confirming the identification of some sand flies, to Prof. Dr Peter Overath from the Max Planck Institute for Biochemistry for providing Leishmania strains, and Jacqueline Dockray for reading the manuscript.

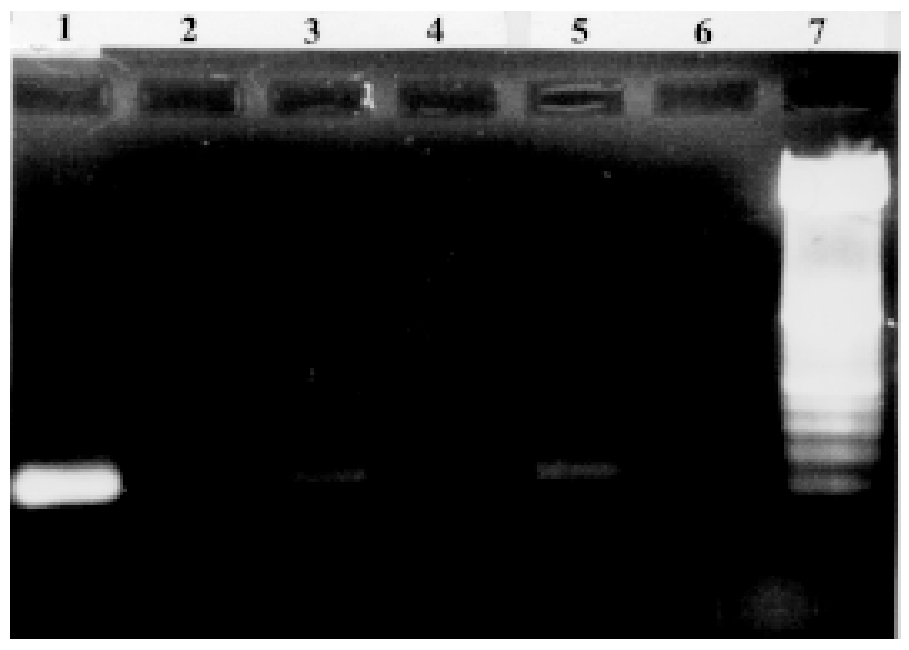

Polymerase chain reaction based detection of natural infection of Phlebotominae with Leishmania (Viannia). 1: positive control (DNA Leishmania (V.) braziliensis); 2: negative control (DNA) Le. mexicana); 3-4: Lutzomyia pessoai; 5: Lu. misionensis, 6: negative control (without DNA); 7: marker. 


\section{REFERENCES}

Arevalo J, Inga R, Lopes M 1993. Polymerase chain reaction. Detection of Leishmania braziliensis, $\mathrm{p}$. 456-46. In DH Persing, FC Penover, T Smith, TJ White (eds), Diagnostic Molecular Microbiology: Principles and Applications, Amer Soc of Microbiol, Washington.

Dias ES, Falcão AL, Silva JE 1997. Notes on the sand fly fauna (Diptera: Psychodidae) in the State of Rio Grande do Sul. Mem Inst Oswaldo Cruz, 92: 329332.

Forattinni OP 1973. Entomologia Médica: Psychodidae. Phlebotominae. Leishmanioses. Bartolenose, $4^{\circ}$ vol., Universidade de São Paulo, São Paulo, 658 pp.

Lacerda MM 1994. The Brazilian leishmaniasis control program. Mem Inst Oswaldo Cruz 89: 489-495.
Martins AV, Willians P, Falcão AL 1978. American Sandflies (Diptera: Psychodidae, Phlebotominae), Academia Brasileira de Ciências, Rio de Janeiro, 195 pp.

Rebollar-Téllez E, Ramírez-Fraire A, Andrade-Narvaes FJ 1996. A two years study on vectors of cutaneous leishmaniasis. Evidence for sylvatic transmission cycle in the state of Campeche, Mexico. Mem Inst Oswaldo Cruz 91: 555-560.

Shannon RC 1939. Methods for collecting and feeding mosquitoes in jungle yellow fever studies. Am J Trop Med 19: 131-140.

Young DG, Duncan MA 1994. Guide to the identification and geographic distribution of Lutzomyia sand flies in Mexico, the West Indies, Central and South America (Diptera: Psychodidae). Mem Amer Entomol Inst 54: 1-881. 